\title{
Roles of Wnt/ $\beta$-catenin signaling in the gastric cancer stem cells proliferation and salinomycin treatment
}

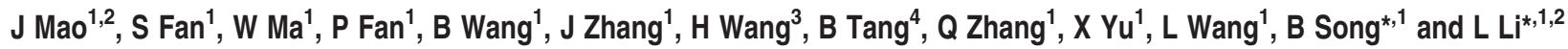

The Wnt1 protein, a secreted ligand that activates Wnt signaling pathways, contributes to the self-renewal of cancer stem cells (CSCs) and thus may be a major determinant of tumor progression and chemoresistance. In a series of gastric cancer specimens, we found strong correlations among Wnt1 expression, CD44 expression, and the grade of gastric cancer. Stable overexpression of Wnt1 increased AGS gastric cancer cells' proliferation rate and spheroids formation, which expressed CSC surface markers Oct4 and CD44. Subcutaneous injection of nude mice with Wnt1-overexpressing AGS cells resulted in larger tumors than injection of control AGS cells. Salinomycin, an antitumor agent, significantly reduced the volume of tumor caused by Wnt1-overexpressing AGS cells in vivo. This is achieved by inhibiting the proliferation of CD44 + Oct4 + CSC subpopulation, at least partly through the suppression of Wnt1 and $\beta$-catenin expression. Taken together, activation of Wnt1 signaling accelerates the proliferation of gastric CSCs, whereas salinomycin acts to inhibit gastric tumor growth by suppressing Wnt signaling in CSCs. These results suggest that Wnt signaling might have a critical role in the self-renewal of gastric CSCs, and salinomycin targeting Wnt signaling may have important clinical applications in gastric cancer therapy.

Cell Death and Disease (2014) 5, e1039; doi:10.1038/cddis.2013.515; published online 30 January 2014

Subject Category: Cancer

Cancer stem cells (CSCs) or cancer-initiating cells (CICs) accounting for a rare fraction of a certain tumor share many properties with somatic stem cells, such as a capacity for selfrenewal and multipotent differentiation potential. ${ }^{1}$ Mounting evidence indicate that CSCs may determine tumorigenesis, invasive growth, metastasis formation, and relapse potential. ${ }^{2}$ CSCs have been identified in a variety of solid tumors, including breast cancer, ${ }^{3}$ glioma, ${ }^{4}$ prostate cancer, ${ }^{5}$ ovarian cancer, ${ }^{6}$ and lung cancer. ${ }^{7}$ Similarly, CSCs were isolated from a panel of gastric cancer cell lines using cell surface marker CD44 in 2009. ${ }^{8}$ The CD44 + gastric cancer cells showed spheroid colony formation under low-attachment culture condition in vitro as well as tumorigenic potential in vivo. Most importantly, the CD44 + gastric cancer cells appeared more resistant to 5-fluorouracil and etoposide (VP-16) treatment and radiotherapy. However, the mechanisms regulating selfrenewal and chemoresistance of gastric CSCs are not fully understood.

Wnts family consisting of 19 secreted glycoproteins is critical to regulate cell proliferation, migration, and differentiation. ${ }^{9}$ Wnt proteins bind to the low-density lipoprotein receptor-related protein $5 / 6$ and Frizzled to block the phosphorylation and degradation of $\beta$-catenin. The $\beta$-catenin then translocates into the nucleus and interacts with T-cell factor/lymphoid-enhancing factor (TCF/LEF) to activate the downstream target genes related with cell proliferation, survival, and progression. ${ }^{10} \mathrm{Wnt} / \beta$-catenin signaling pathway has been implicated in the maintenance of self-renewal in various types of stem cells. ${ }^{11}$ Abnormal activation of Wnt/ $\beta$-catenin signaling pathway strongly correlates with tumorigenesis and progression through maintaining $\mathrm{CICs}^{12} \mathrm{How}-$ ever, this relationship has been studied more extensively in leukemias, colorectal, and mammary cancers than in gastric cancers. ${ }^{13}$ Gastric cancer is currently the second common cancer in China right after lung cancer, and meanwhile it is the second leading cause of cancer death. ${ }^{14}$ Elucidating the role of Wnt signaling in the gastric cancer may help the development of more efficacious treatments.

Salinomycin is the first agent being identified as a selective inhibitor of breast CSCs, and it was able to reduce breast CSCs by more than 100-fold compared with paclitaxel treatment ${ }^{15}$ Aldehyde dehydrogenase (ALDH)-high gastric cancer cells are relatively sensitive to salinomycin compared with ALDH-low cancer cells. ${ }^{16}$ Recently, salinomycin was found to inhibit Wnt/ $\beta$-catenin signaling by inducing the degradation of Wnt co-receptor LRP6 in chronic lymphocytic leukemia. ${ }^{17}$ Tang et al. ${ }^{18}$ reported that salinomycin selectively targets osteosarcoma stem cells possibly by inhibiting the $\mathrm{Wnt} / \beta$-catenin signaling pathway. On the basis of above results, we hypothesized that the inhibitory effect of salinomycin on $\mathrm{Wnt} / \beta$-catenin signaling may cause toxicity to gastric CSCs.

\footnotetext{
${ }^{1}$ Departmemt of Pathology, Dalian Medical University, Dalian 116044, P.R. China; ${ }^{2}$ The Key Laboratory of Tumor Stem Cell Research of Liaoning province, Dalian Medical University, Dalian 116044, P.R. China; ${ }^{3}$ Academic Affair Department of Dalian Medical University, Dalian 116044, P.R. China and ${ }^{4}$ Department of Hepatobiliary Surgery, Affiliated Hospital of Guilin Medical University, Guilin 541001, P.R. China

${ }^{*}$ Corresponding author: B Song or L Li, Department of Pathology, Dalian Medical University, No. 9 West Section, Lvshun South Road, Dalian 116044, P.R. China. Tel/Fax: +86 4118611 0299; E-mail: yr0806@ hotmail.com (BS) or Tel/Fax: +86 4118611 0050; E-mail: lilianhong0051@163.com (LL)

Keywords: Wnt signaling; gastric cancer stem cells; salinomycin

Abbreviations: CSCs, cancer stem cells; CICs, cancer-initiating cells; ALDH, aldehyde dehydrogenase; SP, side population; TCF/LEF, T-cell factor/lymphoidenhancing factor; UC, ulcerative colitis

Received 05.9.13; revised 01.11.13; accepted 04.11.13; Edited by A Stephanou
} 
Table 1 The expression of Wnt1 and CD44 in gastric tissues

\begin{tabular}{|c|c|c|c|c|c|}
\hline & Low (\%) Wnt1/CD44 & Moderate (\%) Wnt1/CD44 & Intense (\%) Wnt1/CD44 & $N$ & $P$-value Wnt1/CD44 \\
\hline Adjacent normal gastric mucosa & $2(9.0) / 3(13.6)$ & $1(4.5) / 2(9.0)$ & $1(4.5) / 1(4.5)$ & 22 & \\
\hline Precancerous lesions & $5(10.9) / 12(33.3)$ & $11(23.9) / 10(21.7)$ & $12(26.1) / 7(15.2)$ & 46 & \\
\hline Early gastric adenocarcinoma & $5(17.9) / 7(25.0)$ & $6(21.4) / 7(25.0)$ & $9(32.1) / 8(28.5)$ & 28 & \\
\hline Advanced gastric adenocarcinoma & $14(23.3) / 7(11.7)$ & $16(26.7) / 21(35.5)$ & $22(37.7) / 20(33.3)$ & 60 & $0.001 / 0.027$ \\
\hline
\end{tabular}

Table 2 The relationship between CD44 and Wnt1 in gastric cancer (early gastric adenocarcinoma and advanced gastric adenocarcinoma)

\begin{tabular}{llllll}
\hline Protein expression & & \multicolumn{4}{c}{ Wnt1 } \\
\cline { 3 - 4 } & $\boldsymbol{N}$ & + & - & $\boldsymbol{r}$ & $\boldsymbol{P}$ \\
\hline CD44 & & & & & \\
+ & 70 & 62 & 8 & 0.875 & 0.000 \\
- & 18 & 12 & 6 & & \\
\hline
\end{tabular}

In this study, we first found a strong relevance between Wnt1 expression and gastric CSCs marker, CD44 expression in a series of samples of normal gastric mucosa, precancerous lesion, early and advanced gastric adenocarcinoma by immunohistochemistry. Then, we stably overexpressed Wnt1 in the AGS gastric cancer cell line and found that the cellular proliferation rate was significantly increased. More importantly, overexpression of Wnt1 dramatically improved the capacity of spheroids formation and enriched the side population (SP) cells with stem-like cell properties. Consistently, CSCs surface marker CD44, CSC-associated Oct4 gene, ${ }^{19,20}$ and the downstream component of Wnt $1, \beta$-catenin were upregulated in Wnt1 stably transfected AGS cells at both mRNA and protein levels. Subcutaneous injection of nude mice with Wnt1-overexpressing AGS cells resulted in larger tumors than injection of control AGS cells, whereas salinomycin significantly reduced the volume in vivo. This is achieved by inhibiting the proliferation of CD44+Oct4+ CSC subpopulation, possibly through the suppression of Wnt1 and $\beta$-catenin expression. These results suggest that Wnt signaling might have a critical role in the self-renewal of gastric CSCs, and salinomycin inhibits gastric cancer by targeting gastric CSCs with elevated Wnt signaling.

\section{Results}

Stem-related genes $W n t 1$ and $C D 44$ are overexpressed in gastric cancer specimens. CSCs are thought to share many features with somatic stem cells, and the prevalence and (or) activity of these CSCs may be positively correlated with the malignant phenotype. We analyzed the expression of Wnt1 and CD44 in different clinical grade of human gastric cancer tissues because Wnt1 and CD44 overexpression has been demonstrated in other CSCs and may be essential for CSCs self-renewal and tumorigenesis. All parameters of immunohistochemical detection are summarized in Tables 1 and 2 and Figure 1. Statistical analysis revealed that the expression of CD44 and Wnt1 was significant in different gastric tissues ( $P=0.001$ and 0.027 , respectively), and the level of Wnt1 expression was association with the expression of CD44 in gastric cancer $(r=0.875, P=0.000)$. As cells strongly expressing Wnt1 and (or) CD44 may represent a 'stem cell-like' population, ${ }^{1}$ these results suggest that CSCs may contribute to the tumorigenesis of human gastric cancer.

Wnt1 overexpression increases AGS cell proliferation. As Wnt1 was overexpressed in the gastric cancer tissues, we subsequently investigated the effect of Wnt 1 on cell proliferation in the gastric cancer AGS cell line. We first transfected Wnt1 plasmid into the AGS cells, and the empty plasmid (Control) and AGS cell alone (N-Control) were used as the negative controls. We found transfection of Wnt1 plasmid for $48 \mathrm{~h}$ had the highest transfection rate than that any other time points when evaluated by fluorescence microscopy (data not shown). The cells were then cultured under antibiotic selection pressure for 21 days and collected to examine the Wnt1 levels by RT-PCR and western blot. Wnt1 was significantly overexpressed at both mRNA and protein levels indicating the successful transfection (Figures $2 \mathrm{a}$ and b). Next, we evaluated the proliferation rate by CCK8 assay. As Figure 2c show, the upregulation of Wnt1 dramatically increased the cell growth compared with its negative controls. A statistical difference was found at 72 and $96 \mathrm{~h}$ after plating, and there was no significant difference between the two negative controls. In order to speculate how Wnt1 overexpression stimulates the cell proliferation, we detected the S-phase percentage in Control, N-Control and AGS-Wnt1 cells by flow cytometry. We found that the S-phase percentage in AGS-Wnt1 cells was much higher than those in the Control and $\mathrm{N}$-Control cells after $96 \mathrm{~h}$ (Figure 2d), which were 18.22 $\pm 3.11,17.44 \pm 2.56$ and $28.16 \pm 4.5 \%\left({ }^{\star \star} P<0.01\right)$. These results demonstrated that Wnt1 overexpression improves the cell growth potential of gastric cancer cell line possibly through increasing the S-phase portion.

Wnt1 enhances the spheroid formation capacity and enriches the percentage of SP cells. Previously we found that Wnt1 was overexpressed along with the progression from normal gastric epithelium to gastric precancerous lesion and gastric cancer. Interestingly, CD44, the gastric CSC marker, was also overexpressed and the Spearman correlation analysis showed there was a significant positive correlation between Wnt1 and CD44 expression. These data indicated that Wnt1 might contribute to the maintenance of gastric CSCs. 


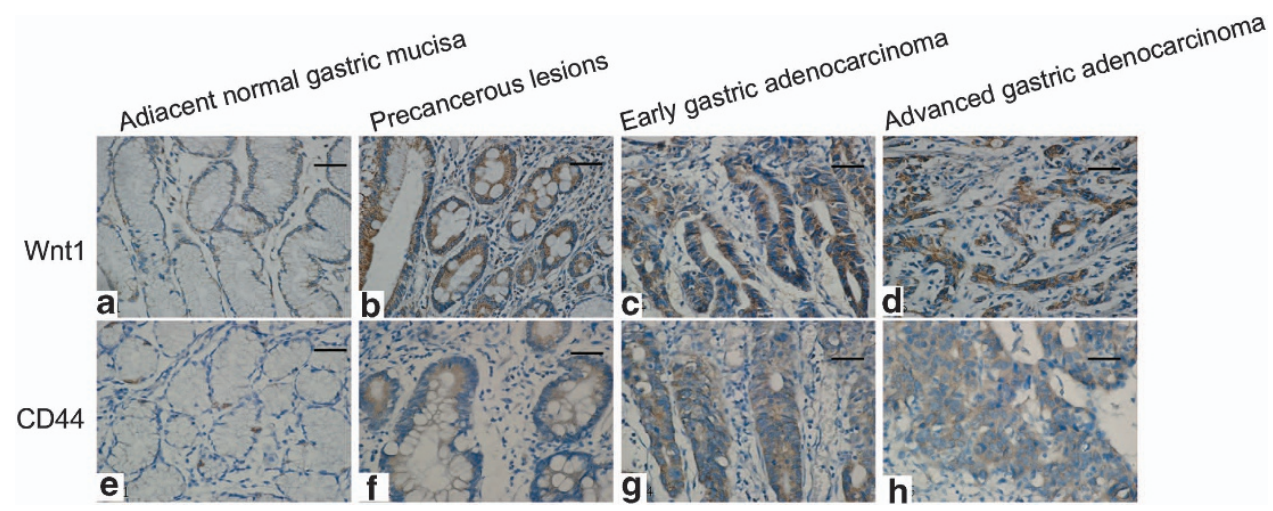

Figure 1 Immunochemical expression of Wnt1 and CD44 in human gastric cancer tissues of different clinical grades. (a-d) The expression of Wnt1. (e-h) The expression of CD44. Scale bar $=50 \mu \mathrm{m}$

a
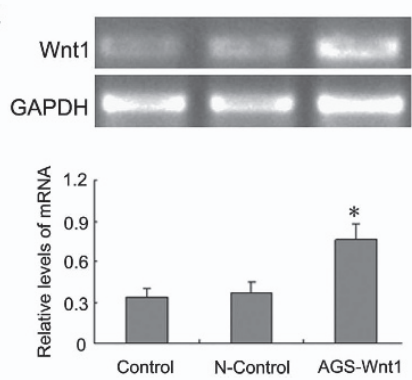

b

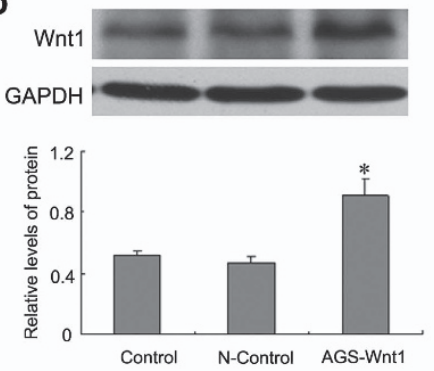

C

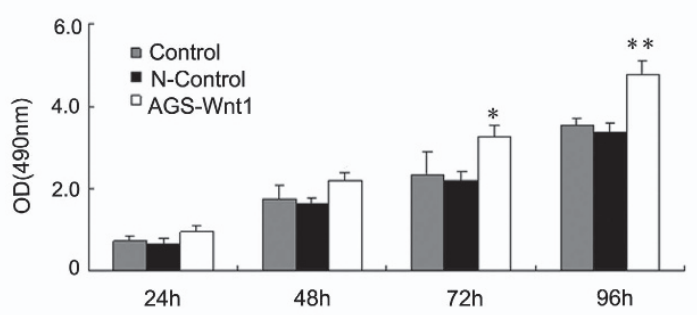

d
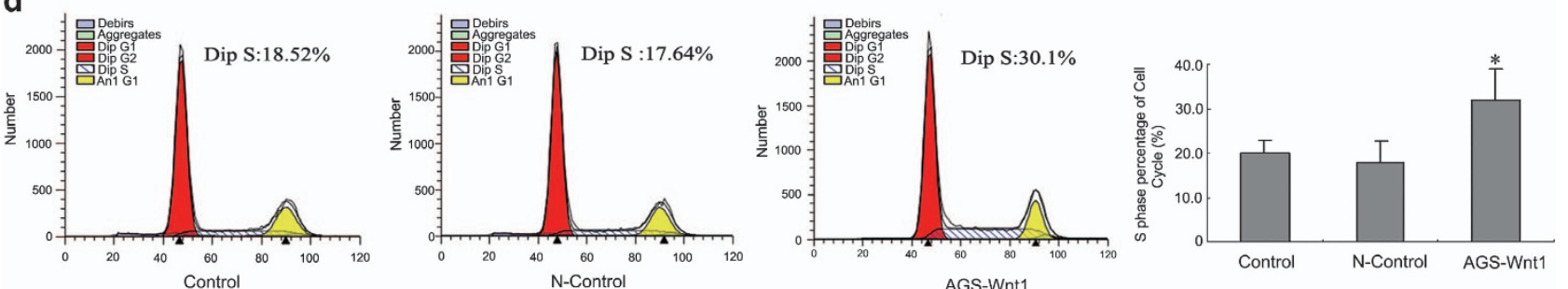

Figure 2 Overexpression of Wnt1 enhanced AGS cell proliferation rate compared with untransfected negative control AGS cells (N-Control) and AGS cells without treated (Control). (a) RT-PCR analysis revealed elevated Wnt1 mRNA expression in the AGS-Wnt1 cell line. ${ }^{\star \star} P<0.01$. (b) Western blot analysis of Wnt1 protein expression. ${ }^{* \star} P<0.01$. (c) Viable cell counting using the CCK8 assay revealed enhanced AGS-Wnt1 cell number at $96 \mathrm{~h}$ after plating. ${ }^{*} P<0.05$. (d) The S-phase percentage was increased significantly in the AGS-Wnt1 cells at $96 \mathrm{~h}$ after plating. ${ }^{* *} P<0.01$. These experiments were repeated three times and error bars represent S.D. The representative flow cytometry pattern is shown

Sphroid formation is a typical property of CSCs that reflects the self-renewal potential. ${ }^{3}$ Therefore, spheroid formation assay was performed here to determine whether Wnt1 can increase the propagation of gastric CSC. AGS cells with or without stably transfected Wnt1 were cultured under ultra-low attachment conditions in serum-free media. After 7 days, about $72.6 \pm 1.28 \%$ of AGS-Wnt1 wells produced spherical colonies compared with only $36.3 \pm 0.98 \%$ of wells inoculated with $\mathrm{N}$-Control cells (Figure $3 \mathrm{a}$; ${ }^{* *} P<0.01$ ). Except the sphroid formation capacity, the CSCs can also be identified by the detection of SP cells by Hoechst 33342 exclusion. SP cells accounting for a small fraction of the whole-cell population including the normal and cancerous settings appear as distinct stem-like properties. We next evaluated whether Wnt1 influences the fraction of SP cells. As shown in Figure 3b, AGS-Wnt1 cells harbored a significantly greater fraction of SP cells than $\mathrm{N}$-Control cells $(7.7 \pm 1.2$ versus $4.2 \pm 0.35 \%$; ${ }^{\star \star} P<0.01$ ).

To confirm the greater prevalence of CSC phenotype within the AGS-Wnt1 population, we also tested the expression levels of CD44 and Oct4 by RT-PCR, western blot, and immunofluorescence. We found that AGS-Wnt1 cells expressed higher levels of CD44 and Oct4 both at mRNA and protein levels (Figures $3 \mathrm{c}$ and d; ${ }^{*} P<0.05$ ). Consistently, the expression of CD44 and Oct4 proteins was also stronger in AGS-Wnt1cells compared with $\mathrm{N}$-Control cells as demonstrated by immunofluorescence (Figure 3e). Furthermore, AGS-Wnt1 cells expressed high levels of $\beta$-catenin, a protein that transduces cytoplasmic Wnt1-initiated signaling cascades into the nucleus (Figures $3 \mathrm{c}$ and d; ${ }^{*} P<0.05$ ). Taken together, these data implied that Wnt1 upregulation can enhance the proliferation potential of gastric CSCs. 
a

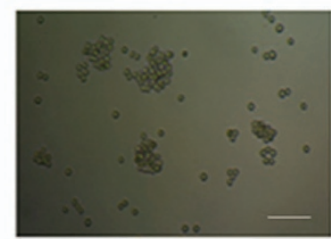

$\mathrm{N}-$ Control

b

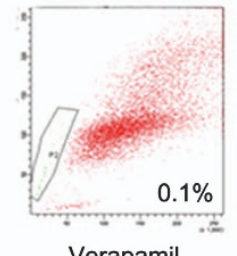

Verapamil

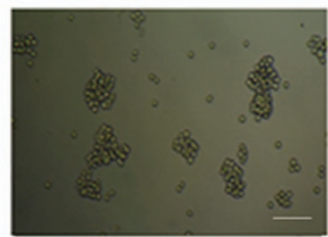

AGS-Wnt1

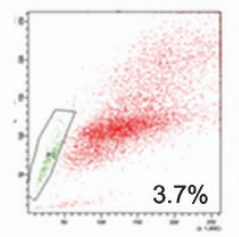

N-Control

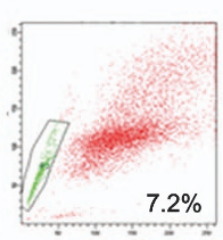

AGS-Wnt1
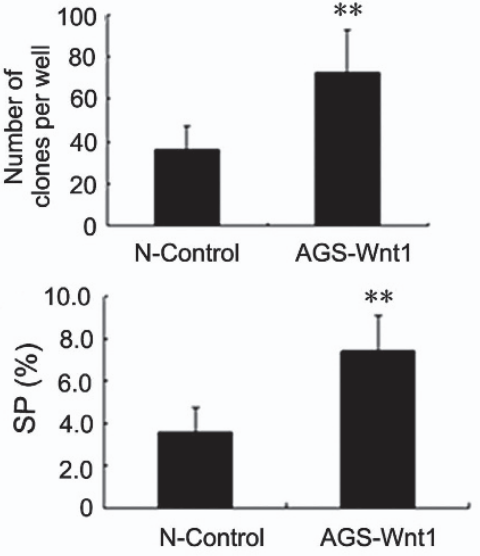

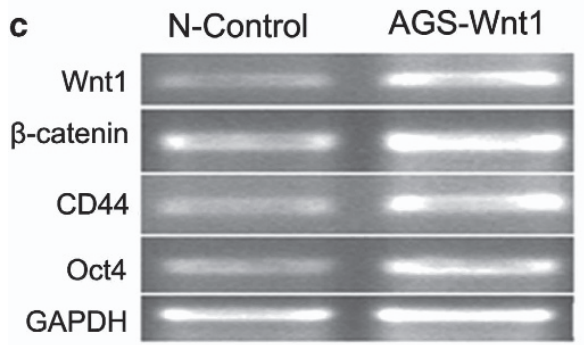

d

N-Control AGS-Wnt1
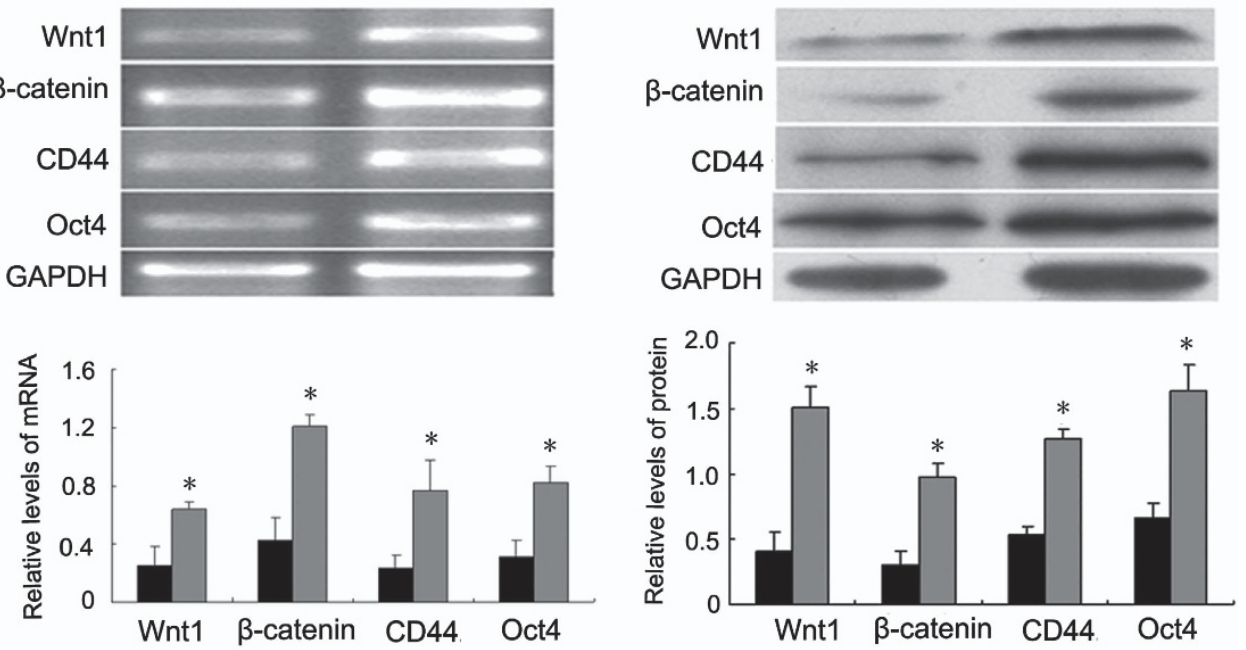

e
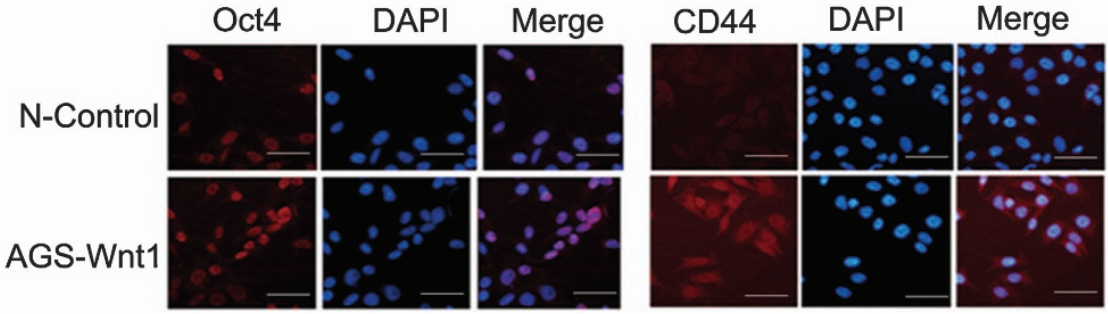

Figure 3 Wnt1 enhances the spheroid formation capacity and enriches the percentage of SP cells. (a) AGS cells with or without stably transfected Wnt1 were cultured under ultra-low attachment conditions in serum-free media for 7 days. Spherical colonies were collected and evaluated (scale bar $=100 \mu \mathrm{m}$ ). ${ }^{* \star} P<0.01$. (b) The percentage of SP cell by Hoechst 33342 exclusion was analyzed using flow cytometry. Similar results were obtained in three independent experiments, and the representative microscopic picture and flow cytometry pattern are shown. ${ }^{* *} P<0.01$. (c and d) As determined by RT-PCR and western blot, the expression levels of Wnt1, $\beta$-catenin, Oct4, and CD44 mRNAs and proteins were elevated in AGS-Wnt1 cells compared with N-Control. ${ }^{*} P<0.05$. (e) Immunofluorescent staining of CD44 and Oct4 in cultured N-Control and AGS-Wnt1 cells confirmed higher CD44 and Oct4 protein expressions in AGS-Wnt1 cells (scale bar $=50 \mu \mathrm{m}$ )

Wnt1 knockdown inhibits the proliferation potential of AGS cells and gastric CSCs. To test whether we can reverse the effects caused by exogenous Wnt1, we transfected AGS-Wnt1 cells with $100 \mathrm{nM}$ of siRNA against Wnt1. AGS-Wnt1 cells alone (AGS-Wnt1) and scrambling nucleotide sequence of siRNA ( $\mathrm{N}$-Control) were used as the negative controls. We found that siRNA against Wnt1 downregulated the expression of Wnt1 at both mRNA and protein levels when compared with the negative controls
(Supplementary Figures $\mathrm{A}$ and $\left.\mathrm{B} ;{ }^{*} P<0.05\right)$, indicating the successful transfection of siRNA. Then we detected the effects of Wnt1 knockdown on the cellular proliferation by CCK8 and colony formation assays. As shown in Supplementary Figure $\mathrm{C}$, the proliferation rate of AGSWnt 1 cells was dramatically inhibited by knockdown of Wnt1, and the differences were found statistically significant from 48 to $96 \mathrm{~h}$ after transfection with siRNA $\left({ }^{*} P<0.05\right)$. For the colony formation assay, we counted colonies containing at 
A

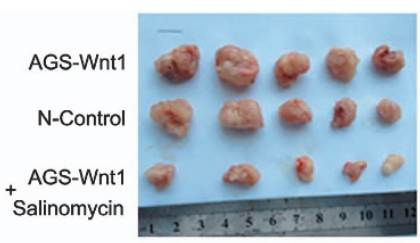

C
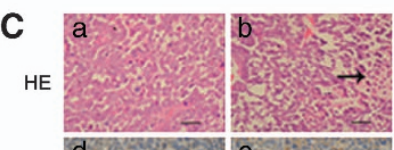

CD44
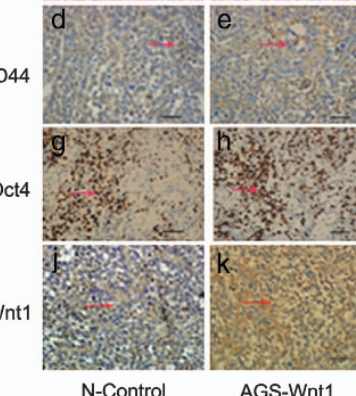
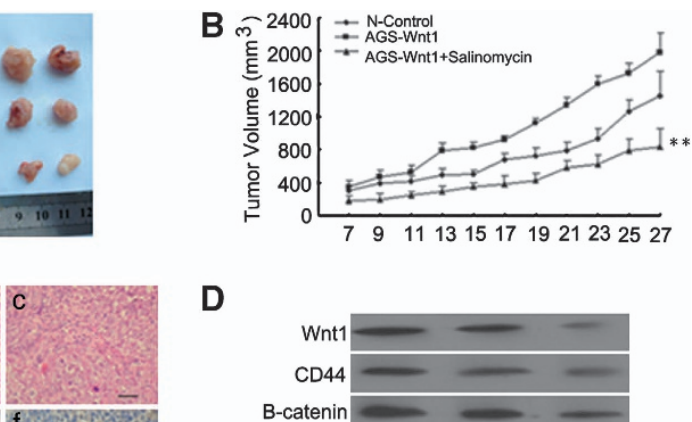

D

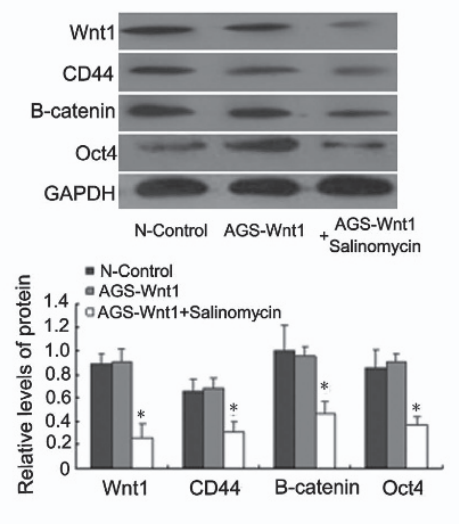

Figure 4 Wnt1 overexpression enhances the tumorigenicity and CSC phenotype expression that is inhibited by salinomycin in nude mice. (A) A photograph of peripheral tumors after excision on day 28 post inoculation. AGS-Wnt1 cells formed larger tumors than $\mathrm{N}$-control cells, whereas subsequent salinomycin treatment significantly reduced the tumor mass in the AGS-Wnt1-injected group (scale bar $=1 \mathrm{~cm}$ ). (B) The volume of the peripheral tumor was also estimated in vivo every 2 days post inoculation. Columns, mean volume of each group at the corresponding time point. ${ }^{* *} P<0.01$ compared with the $\mathrm{N}$-Control group at the corresponding time. (C) HE and immunohistochemical staining with CD44 and Oct4 antibodies. (a-d) HE staining. (e-i) Immunostaining for CD44, Oct4, and Wnt1 (brown color in cytoplasm), $(\times 200$; scale bar $=100 \mu \mathrm{m}$ ). (D) Protein expression levels and analysis of Wnt1, $\beta$-catenin, Oct4, and CD44 in the xenograft tumors. GAPDH was used as the internal control. ${ }^{*} P<0.05$

least 50 cells on day 7 after plating. We found that siRNA against Wnt1 significantly suppressed the colony-forming capacity when compared with the two negative controls (Supplementary Figure D). These results demonstrated that Wnt1 downregulation can inhibit the cell growth potential of gastric cancer cell line.

To further confirm the impact of Wnt1 on the self-renewal of gastric CSC, we detected the percentage of SP cell by Hoechst 33342 exclusion at $7 \mathrm{~d}$ after antagonizing Wnt 1 in AGS-Wnt1 cells. As shown in Supplementary Figure E, the fraction of SP cell was reduced from $6.8 \pm 1.2$ to $3.0 \pm 0.8 \%$ after downregulating Wnt1, and the difference was statistically significant compared with AGS-Wnt1 cells alone $\left({ }^{* *} P<0.01\right)$. These data further demonstrated that Wnt1 has an important role in the proliferation of gastric cancer cells and CSCs.

Wnt1 enhances the tumorigenic behavior of gastric cancer in vivo that is inhibited by salinomycin. Given the observed effects of Wnt1 on gastric cancer cell proliferation in vitro, we subsequently determined whether Wnt1 enhances tumorigenicity in vivo. We compared tumor growth following subcutaneous injection of $\mathrm{N}$-Control or AGS-Wnt1 cells in nude mice and found that at time points, tumors in the AGS-Wnt1-injected group were markedly larger than those in the $\mathrm{N}$-Control injected group, whereas subsequent treatment with salinomycin significantly reduced the tumor mass in the AGS-Wnt1-injected group (Figures 4A and B).

Next, we assessed the capacity of Wnt1 to inhibit gastric CSC proliferation in vivo. We used immunohistochemistry to detect the expression of CD44, Oct4, and Wnt1 in the mouse xenograft tumor tissues. As shown in Figure 4C, CD44, Oct4, and Wnt1 were highly expressed in AGS-Wnt1 group compared with $\mathrm{N}$-Control group. The expressions of Wnt 1 and CSC markers, CD44 and Oct4 appear as the same tendency, although no relevance was found $(P>0.05)$. We also measured the levels of CD44 and Oct4 by western blot in the mouse tumor tissues. CD44 and Oct4 proteins were significantly upregulated by Wnt1 (Figure 4D). Consistently, $\beta$-catenin was increased while Wnt1 was overexpressed (Figure 4D). These results indicated that Wnt1 upregulation improves the growth of gastric cancer cell in vivo and enriches the CD44- and Oct4-positive cell population in the xenograft tumors.

We then investigated the inhibitory mechanism of salinomycin in vivo. The expression of stemness markers, CD44 and Oct4 proteins, was seen to be reduced dramatically in the AGS-Wnt1-injected xenograft tumor tissues with salinomycin treatment on immunohistochemical analysis (Figure 4C). Western blot analysis also showed that salinomycin treatment suppressed the expression levels of CD44 and Oct4. Furthermore, the protein levels of Wnt1 and $\beta$-catenin were decreased by salinomycin (Figure 4D). Therefore, salinomycin inhibits the growth of gastric cancer cells in vivo, likely by decreasing the proliferation of a CD44/Oct4-positive stem cell-like population by targeting $\mathrm{Wnt} / \beta$-catenin signaling.

\section{Discussion}

CSCs representing a minor subpopulation of tumor cells have been thought to play a major role in tumor initiation and 
resistance to chemotherapy and radiation therapy. ${ }^{21}$ Various molecular signaling pathways including $\mathrm{Wnt} / \beta$-catenin are identified to be involved in maintaining CSCs and thus are responsible for the recurrence and poor prognosis of tumor. ${ }^{12}$ In this study, we investigated the role of Wnt signaling in gastric CSC self-renewal, its clinical relevance, and the relationship with the toxicity of salinomycin to gastric cancer.

We initially observed that Wnt1 was overexpressed in a graded manner in a series of normal gastric mucosa, precancerous lesion, and early and advanced gastric adenocarcinoma specimens, and there was a strong positive relevance between Wnt1 expression and gastric CSCs marker, CD44 expression by immunohistochemistry. We then stably transfected Wnt1 in the gastric cancer AGS cells and found that Wnt1 enhanced the cell proliferation potential and in the meantime increased the portion of $S$ phase in vitro. Wnt1/ $\beta$-catenin signaling has been reported to control G1/S transition by regulating cyclin D1 transcription. ${ }^{22,23}$ Rimerman et al. ${ }^{24}$ found that Wnt1 cooperates with MEK1 to promote cyclin D1 accumulation. Megason et al. ${ }^{25}$ reported that Wnt/ $\beta$-catenin pathway positively regulates cell cycle progression partly through transcriptional regulation of cyclin D1 and cyclin D2. Thus, Wnt1 stimulates the cell proliferation potential by increasing the percentage of $S$ phase, and this is achieved possibly by the accumulation of cyclin D1 and D2. More importantly, overexpression of Wnt1 dramatically improved the capacity of spheroids formation and enriched the SP cells with stem-like cell properties. Consistently, CSCs markers, Oct4 and CD44, and the downstream component of Wnt1, $\beta$-catenin, were upregulated in the Wnt1 stably transfected AGS cells at both mRNA and protein levels. Thus, a highly tumorigenic subpopulation of CSCs is indeed present in AGS cells and Wnt 1 overexpression increases the relative prevalence of these cells. In order to prove our results, we transfected siRNA against Wnt1 into AGS-Wnt1 cells (siRNA-AGS-Wnt1) to silence Wnt1 gene expression. We found that the proliferation rate was dramatically inhibited by knockdown of Wnt1 (Supplementary Figures C and D; ${ }^{\star} P<0.05$ ). Meanwhile, as shown in Supplementary Figure $E$, the percentage of SP cell was reduced from $6.8 \pm 1.2$ to $3.0 \pm 0.8 \%$ after downregulating Wnt1 $\left({ }^{\star *} P<0.01\right)$. These data further demonstrated that Wnt1 has an important role in the cellular proliferation of AGS cells and gastric CSCs.

Subcutaneous injection of Wnt1-overexpressing AGS cells into nude mice resulted in larger tumors than did injection with control AGS cells. CD44 and Oct4 were highly expressed in the mouse xenograft tumors generated from AGS-Wnt1 cells. Our results demonstrated the essential role of $\mathrm{Wnt} / \beta$-catenin signaling in the self-renewal of gastric CSC. This is consistent with another recent study. ${ }^{26}$ In addition, $\mathrm{Wnt} / \beta$-catenin signaling also has a pivotal role in the growth and maintenance of colon CSCs. Kanwar et al. ${ }^{27}$ found that colonospheres derived from the colon cancer cell lines exhibit increased levels of total $\beta$-catenin and reduced phosphorylated $\beta$-catenin. Increased expression of $\beta$-catenin can induce the transcriptional activation of TCF/LEF and $\beta$-catenin silence by siRNA leads to decreased colonosphere formation. Vermeulen et al. $^{28}$ reported that colon CSCs exhibit high activity of $\mathrm{Wnt} / \beta$-catenin signaling using a Wnt reporter construct. Corbo et al. ${ }^{29}$ demonstrated that colon CSCs with
CD133 + express a higher $\mathrm{Wnt} / \beta$-catenin pathway when compared with CD133 population by mass spectrometry. Interestingly, Shenoy et al. found that ulcerative colitis (UC), a precancerous lesion of colorectal cancer, shows an intermediate level of Wnt-pathway-active cells between normal colon and colorectal cancer. These UC cells with active Wnt pathway constitutes a major component of the colonic epithelial cells that are positive for ALDH. ALDH $+U C$ cells with the highest Wnt activity present as higher clonogenic and tumorigenic potential than those with the lowest Wnt activity, indicating the critical role of Wnt activity in driving CSC-like properties in these cells. ${ }^{30}$ These data highlight the role of $\mathrm{Wnt} / \beta$-catenin signaling not only in the growth and maintenance of gastrointestinal tract CSCs but also in the progressive process from the precancerous lesion to the cancer through driving CSCs.

The Oct4 gene, a POU family transcription factor, has a critical role in the self-renewal of embryonic stem (ES) cells and adult stem cells. ${ }^{31,32}$ However, continuous Oct4 expression was reported to induce the dysplastic growth of mice epithelial cells in the GI tract and skin. The authors further proved that the target cells of Oct4-induced dysplasia are stem cells and Oct4 can expand the progenitor cells in dysplastic lesions. ${ }^{33}$ High levels of Oct4 also increase the malignant potential of ES-derived tumors, whereas inactivation of Oct4 reduces malignancy. ${ }^{34}$ Oct4 is already found to be expressed in the breast cancer cell lines and samples, ${ }^{35-37}$ colon cancer cell line,${ }^{38}$ and bladder cancer. ${ }^{39}$ Very interestingly, CSCs in some types of human solid tumors, such as breast, ${ }^{36}$ gastric, ${ }^{40}$ and osteosarcoma ${ }^{18}$ overexpress Oct4. $\mathrm{Hu}$ et $a .^{36}$ demonstrated that siRNA against Oct4 successfully reduces the CSC-like cells and inhibits the tumor growth. These reports indicate that Oct4 is critical not only in the stem cells but also in the CSCs. Kelly et al. ${ }^{41}$ found that stabilized $\beta$-catenin can bind with and attenuate the activity of Oct4 and therefore regulate the pluripotency. Another report indicated that $\beta$-catenin/CBP/Oct 4 complex may regulate the functional switching of $\beta$-catenin. ${ }^{42}$ Hochedlinger et al. ${ }^{43}$ also found that ES cell-derived intestinal crypts express higher levels of $\beta$-catenin compared with the host-derived crypts. Our present study showed that $\mathrm{Wnt} / \beta$-catenin signaling has a critical role in the self-renewal of gastric CSC in vitro and in vivo. Meanwhile, Oct4 was upregulated in the Wnt1 stably transfected AGS cells and in the mouse xenograft tumors generated from AGS-Wnt 1 cells. Thus, Wnt $/ \beta$-catenin signaling may regulate the self-renewal of gastric CSC by increasing the expression of the stemness marker Oct4.

In addition to enhancing the tumorigenicity, previous studies have shown that Wnt1 expression also confers drug resistance to gastric cancers. ${ }^{44,45}$ In our study, we found that salinomycin markedly decreased the growth rate of tumors originating from AGS-Wnt1 cells in nude mice and meanwhile reduced the CD44/Oct4-positive stem cell-like population. The expressions of Wnt 1 and $\beta$-catenin in the xenograft tumor tissues were inhibited as well. Several reports have demonstrated that salinomycin selectively targets CSCs through inhibiting $\mathrm{Wnt} / \beta$-catenin signaling pathway in the chronic lymphocytic leukemia and osteosarcoma. ${ }^{17,18}$ Our results further confirmed that salinomycin inhibits the tumor growth by preferentially targeting the gastric CSCs instead of the entire 
cell population. We also showed that this inhibition is achieved possibly by targeting $\mathrm{Wnt} / \beta$-catenin signaling.

Taken together, we have shown that $\mathrm{Wnt} / \beta$-catenin signaling improves the proliferation of gastric CSCs in vitro and in vivo. We have identified salinomycin as an effective inhibitor of gastric CSCs in vivo possibly by suppressing $\mathrm{Wnt} / \beta$-catenin signaling in CSCs. These results suggest that $\mathrm{Wnt} / \beta$-catenin signaling might have an essential role in the self-renewal of gastric CSCs, and salinomycin targeting $\mathrm{Wnt} / \beta$-catenin signaling may have important clinical applications in gastric cancer therapy.

\section{Materials and Methods}

Patients and tissue specimens. Formalin-fixed and paraffin-embedded gastric malignant and nonmalignant tissue samples were obtained from 156 patients ( 86 males and 68 females) by transurethral gastric tumor resection and radical cystectomy at the First Affiliated Hospital of Dalian Medical University (Dalian, P.R. China) between 2011 and 2013. The mean age was 55 years (range, 35-69). Of the total case series, 22 were normal gastric mucosa and 46 were gastric precancerous lesion (chronic atrophic gastritis with intestinal metaplasia) Twenty-eight and 60 were early and advanced gastric adenocarcinoma respectively. All the tissue slides were routinely stained with hematoxylin and eosin and observed by two independent pathologists according to the WHO criteria (2004). All the samples were obtained after the patients signed the informed consent. The study was approved by the ethics board of the First Affiliated Hospital of Dalian Medical University (Dalian, P.R. China).

Immunohistochemistry. Formalin-fixed and paraffin-embedded gastric malignant and nonmalignant tissue sections were incubated with rabbit polyclonal antibody anti-Wnt1 (dilution 1:200, Santa Cruz Biotechnology, Santa Cruz, CA, USA) and antibody anti-CD44 (dilution 1:200, Santa Cruz Biotechnology) overnight at $4{ }^{\circ} \mathrm{C}$ in a humidified container. Detection was determined by nonbiotin horseradish peroxidase detection system and DAB substrate (Dako, Carpinteria, CA, USA). Immunostaining was scored by two independent observers. Briefly, five random views of stained sections were chosen and total 100 tumor cells per view were counted for analysis. Expression of Wnt1 or CD44 in less than $10 \%$ of tumor cells examined was defined as negative, expression in $10-29 \%$ was defined as low expression, $30-60 \%$ was defined as moderate expression, and $>60 \%$ was defined as intense expression. ${ }^{46}$

Cell culture and reagents. The human gastric cancer cell line AGS was purchased from the American Type Culture Collection (Manassas, VA, USA) and was maintained in RPMI-1640 medium (Gibco Laboratories, Grand Island, NY, USA) at $37^{\circ} \mathrm{C}$ in a humidified incubator with $5 \% \mathrm{CO}_{2}$. The culture medium was supplemented with $10 \%$ fetal bovine serum (Gibco Laboratories), $2 \mathrm{mmol} / \mathrm{l}$ glutamine, $100 \mathrm{U} / \mathrm{ml}$ penicillin, and $100 \mathrm{mg} / \mathrm{ml}$ streptomycin (Invitrogen, Carlsbad, CA, USA). Salinomycin was purchased from Sigma (St Louis, MO, USA).

Cell transfection. The pGPU6/GFP/Neo-Wnt1 and empty pGPU6/GFP/Neo plasmids were obtained from GenePharma (Shanghai, China). AGS cells were transfected with the pGPU6/GFP/Neo-Wnt1 plasmid (AGS-Wnt1) or the empty pGPU6/GFP/Neo vector (N-Control) using the So-fast transfection reagent (GenePharma) following the manufacturer's instructions at a final concentration of $100 \mathrm{nM}$ for $48 \mathrm{~h}$. siRNA against Wnt1 was transfected into AGS-Wnt1 cells (siRNA-Wnt1) to silence Wnt1 gene expression at a final concentration of $100 \mathrm{nM}$, and the following sequences were synthesized using Silencer siRNA (Ambion, Austin, TX, USA): the Wnt1 target sequence $5^{\prime}$-AAGACCTGCTGGATGCGGCT G- $3^{\prime}$, the siRNA sense strand $5^{\prime}$-GACCUGCUGGAUGCGGCUGTT- $3^{\prime}$, and the antisense strand $5^{\prime}$-CAGCCGCAUCCAGCAGGUCTT-3'. Transfection of siRNA duplexes was performed with Oligofectamine reagent according to the manufacturer's guidelines (Jima, Shanghai, China). Scrambling nucleotide sequence of Wnt1 (GenePharma) was used as a negative control (N-Control). Transfection rate was monitored with fluorescence microscopy. The successfully transfecting cells were selected by additional $350 \mathrm{mmol} / \mathrm{l}$ of G418 (Gibco Laboratories). At day 21, cells were collected for RT-PCR and western blot analysis. The cells without transfection were used as a blank control (Control).
CCK8 viable cell counting assay and flow cytometry analysis of cell cycle. Cells were plated in 96-well plates at $3 \times 10^{4}$ cells per well. At 24 , 48, 72, and $96 \mathrm{~h}$ post plating, $10 \mu \mathrm{l}$ Cell Counting Assay Kit-8 solutions (CCK8, Sigma) was added to each well and incubated for $2 \mathrm{~h}$. The absorbance at $490 \mathrm{~nm}$ was measured on a microplate reader. Cells treated with $10 \mu \mathrm{l} \mathrm{RPMl-1640} \mathrm{instead}$ of CCK8 solution were used as the black control. All experiments were repeated in triplicate.

Cells were washed with phosphate-buffered saline (PBS) and fixed in ice-cold $70 \%$ ethanol for $30 \mathrm{~min}$ at $4^{\circ} \mathrm{C}$. Following centrifugation, the cells were resuspended in PBS and stained with $0.05 \mathrm{mg} / \mathrm{ml}$ propidium iodide for $30 \mathrm{~min}$ at $37^{\circ} \mathrm{C}$. The cell suspension was then analyzed on a FACS Calibur cytometer (BD Biosciences, San Jose, CA, USA). Approximately $1 \times 10^{6}$ cells per sample were analyzed.

Colony formation assay. Cells were trypsinized, counted, and seeded for colony formation assay in six-well plates at 300 cells per well. During colony growth, the culture medium was replaced every 3 days. At 7 days after seeding, the colonies were stained with $0.02 \%$ crystal for $1 \mathrm{~h}$ and then were counted in five random chosen fields under an inverted phase-contrast microscope (Olympus IX71; Olympus Corporation, Tokyo, Japan). The colony was counted only if it contained more than 50 cells. Each treatment was carried out in triplicate.

Spheroid formation assay. AGS-Wnt1 cells were cultured in 100-200 $\mu$ of RPMI-1640 medium (Invitrogen) supplemented with $10 \mathrm{mM}$ HEPES (Sigma), $20 \mathrm{ng} / \mathrm{ml}$ human recombinant epidermal growth factor (Invitrogen), and $10 \mathrm{ng} / \mathrm{ml}$ human recombinant basic fibroblast growth factor (Invitrogen). Cell viability was assessed at multiple time points by trypan blue staining (Sigma). After 11 days in vitro, cultures were examined using a light microscope and number of spheroid colonies per well was counted. Each colony contained $>20$ cells.

\section{Detection of SP cells by flow cytometry detection and sorting of} SP cells by FCM. Cells were digested with $0.25 \%$ trypsin-EDTA and then centrifuged for $5 \mathrm{~min}$ at 1000 r.p.m. The cells were subsequently suspended in PBS containing 5\% FBS, and then stained with Hoechst 33342 at a concentration of $10 \mu \mathrm{g} / \mathrm{ml}$, and incubated for $90 \mathrm{~min}$ at $37^{\circ} \mathrm{C}$ either alone or with $100 \mu \mathrm{M}$ verapamil. During the incubation, the cells were shaken every $10 \mathrm{~min}$. A second round of centrifugation was performed for 5 min at 1000 r.p.m., and then the cells were suspended in PBS with $5 \%$ FBS at a concentration of $1 \times 10^{6} \mathrm{cells} / \mathrm{ml}$. The solution was poured through a 400-mesh screen filter and then stored in the dark at $4{ }^{\circ} \mathrm{C}$. The Hoechst 33342 dye was excited at $355 \mathrm{~nm}$ and its dual wavelength fluorescence was analyzed (blue, $450 \mathrm{~nm}$; red, $675 \mathrm{~nm}$ ).

RNA extraction and RT-PCR assay of Wnt1, $\beta$-catenin, CD44, and Oct4. Cells were collected and total RNA was extracted using the RNeasy Total RNA system (Qiagen GmbH, Hilden, Germany) following the manufacturer's protocols. The first-strand cDNA was synthesized using the Reverse Transcriptase System (Invitrogen), and the target cDNAs were amplified using primer pairs for Wnt1, $\beta$-catenin, CD44, and Oct4. GAPDH was used as the internal standard. All the reverse transcription primers above were purchased from Applied Biosystems (Carlsbad, CA, USA). The sequences were Wnt1 forward, 5'-CGCTCTCTTCCAGTTCTCAGACAC-3' and reverse, 5' ${ }^{\prime}$-CAGGATGGCA AAAGGGTTCG- $3^{\prime} ; \beta$-catenin forward, $5^{\prime}$-GCTGATTTGATGGATGGAGTTGG-3' and reverse, 5'-CTACTTGTTCTTGAGTGAA-3'; CD44 forward, 5'-CTCCGGA CACCATGGACAAGT-3' and reverse, $5^{\prime}$-CTCTTCTTATGCTATA-ACCTG- $3^{\prime}$; Oct4 forward, 5'-TGGAGAAGGAGAAGCTGGAGCAAAA-3' and reverse, 5'-GGCAGATGGTCGTTTGGCTGAATA-3'; and GAPDH forward, 5'-ATGTCGTG GAGTCTACTGGC- $3^{\prime}$ and reverse, $5^{\prime}$-TGACCTTGCCCACAGCCTTG- $3^{\prime}$.

Aliquots $(8 \mu \mathrm{l})$ of the amplified products were loaded in the $1.5 \%$ agarose gels, separated by electrophoresis, and visualized by ethidium bromide staining

Western blot analysis. Cells were lysed in $1 \times$ RIPA buffer (Sigma). The protein concentration was determined by Bradford method with BSA (Sigma) as the standard. Equal amount of protein extract $(50 \mu \mathrm{g})$ were subjected to $12 \%$ SDS-PAGE and transferred to PVDF membrane (Millipore, Boston, MA, USA). Blots were labeled with the primary antibodies including Wnt1 $(1: 500), \beta$-catenin (1:400), Oct4 (1:500), and CD44 (1:1000). GAPDH (1:1000) was used as a loading control. The protein bands were visualized using an imaging system (Bio-Rad, Hercules, CA, USA). 
Immunofluorescence. Cells were fixed with $4 \%$ paraformaldehyde, blocked with 10\% normal goat serum (Gibco Laboratories), and incubated with antibody anti-CD44 (dilution 1:100) or anti-Oct4 (dilution $1: 100$ ) overnight at $4{ }^{\circ} \mathrm{C}$ in a humidified container. Immunolabeling was revealed by PE-conjugated secondary antibodies. Nuclei were counterstained with DAPI. Immunofluorescence was observed under a laser scanning confocal microscope (Leica, Solms, Germany).

Tumor growth and morphological analysis in vivo. Fifteen 6- to 8-week-old (18-22 g) nude mice were purchased from the Experimental Animal Center of Dalian Medical University. Mice were randomly divided into two groups. Group 1 containing 5 mice was injected with AGS cells alone (N-Control) and group 2 containing 10 mice was injected with Wnt1-transfected AGS cells (AGS-Wnt1). To each group, $0.5 \times 10^{5}$ cells were resuspended in $100 \mu \mathrm{l}$ of PBS and injected subcutaneously into the dorsa. Five mice were randomly chosen from group 2 and treated with $50 \mathrm{mg} / \mathrm{kg}$ salinomycin every 2 days starting from $7 \mathrm{~d}$ after injection. The tumor mass was monitored using caliper and the tumor volume $(V)$ was calculated using the formula $V=L \times W^{2} \times 0.5$. On day 28 post inoculation, mice were weighed and killed by cervical dislocation.

The xenograft tumors were resected, fixed in $10 \%$ phosphate-buffered formalin, embedded in paraffin, sectioned, and processed for $\mathrm{H} \& \mathrm{E}$ staining. For immunohistochemistry, the tissue sections were incubated with antibody antiCD44 (dilution $1: 200$ ), anti-Wnt1 (dilution $1: 200$ ), and mouse monoclonal antibody anti-Oct4 (dilution $1: 100$ ). The procedures and evaluation of immunostaining were briefly described in the section of Immunohistochemistry. All studies involving mice were approved by the Animal Care and Use Committee of Dalian Medical University.

Statistical analysis. The correlation between the expression levels of CD44 and Wnt1 and clinical grade was analyzed by Fisher's exact test. The relationship between CD44 and Wnt1 in gastric cancer was analyzed by the Spearman test. The results were expressed as mean \pm S.E.M. Analysis was performed using standard one-way analysis of variance (ANOVA) or one-way ANOVA for repeated measures. Statistical significance was set as $P<0.05$.

\section{Conflict of Interest}

The authors declare no conflict of interest.

Acknowledgements. This work was supported by grants from the National Natural Science Foundation of China (No. 81272430 to Lianhong Li and No. 81172052 to Bo Song), from Tumor Stem cell Research Key Laboratory of Liaoning Province to Lianhong Li, and from 'Yingcai' program of Dalian Medical University to Bo Song.

1. Clarke MF, Fuller M. Stem cells and cancer: two faces of eve. Cell 2006; 124: 1111-1115.

2. Tirino V, Desiderio V, Paino F, Rosa AD, Papaccio F, Noce ML et al. Cancer stem cells in solid tumors: an overview and new approaches for their isolation and characterization. FASEB J 2013; 27: 13-24.

3. Al-Hajj M, Wicha MS, Benito-Hernandez A, Morrison SJ, Clark MF. Prospective identification of tumorigenic breast cancer cells. PANS 2003; 100: 3983-3988.

4. Singh SK, Clarke ID, Terasaki M, Bonn VE, Hawkins C, Squire J et al. Identification of a cancer stem cell in human brain tumors. Cancer Res 2003; 63: 5821-5828.

5. Collins AT, Berry PA, Hyde C, Stower MJ, Maitland NJ. Prospective identification of tumorigenic prostate cancer stem cells. Cancer Res 2005; 65: 10946-10951.

6. Bapat SA, Mali AM, Koppikar CB, Kurrey NK. Stem and progenitor-like cells contribute to the aggressive behavior of human epithelial ovarian cancer. Cancer Res 2005; 65 : 3025-3029.

7. Eramo A, Lotti F, Sette G, Pilozzie E, Biffoni M, Virgilo AD et al. Identification and expansion of the tumorigenic lung cancer stem cell population. Cell Death Differ 2008; 15 : 504-514.

8. Takaishi S, Okumura T, Tu S, Wang SS, Shibata W, Vigneshwaran R et al. Identification of gastric cancer stem cells using the cell surface marker CD44. Stem cells 2009; 27: 1006-1020.

9. King TD, Suto MJ, Li YH. The Wnt/ $\beta$-Catenin signaling pathway: a potential therapeutic target in the treatment of triple negative breast cancer. J Cell Biochem 2012; 113: 13-18.

10. Polakis P. The many ways of Wnt in cancer. Curr Opin Genet Dev 2007; 17: 45-51.

11. Reya T, Clevers H. Wnt signalling in stem cells and cancer. Nature 2005; 434 : 843-850.

12. Nguyen LV, Vanner R, Dirks P, Eaves CJ. Cancer stem cells: an evolving concept. Nat Rev Cancer 2012; 12: 133-143.
13. Howe LR, Brown AM. Wnt signaling and breast cancer. Cancer Biol Ther 2004; 3: 36-41.

14. Ling $L$. The incidences and mortalities of major cancers in China. World $J$ Gastroenterol 2006; 12: 17-20.

15. Gupta PB, Onder TT, Jiang G, Tao K, Kuperwasser C, Weinberg RA et al. Identification of selective inhibitors of cancer stem cells by high-throughput screening. Cell 2009; 138 : 645-659.

16. Zhi QM, Chen XH, Ji J, Zhang JN, Li JF, Cai $Q$ et al. Salinomycin can effectively kill ALDH high stem-like cells on gastric cancer. Biomed Pharmacother 2011; 65: 509-515.

17. Lu D, Choi MY, Yu J, Castro JE, Kipps TJ, Carson DA et al. Salinomycin inhibits Wnt signaling and selectively induces apoptosis in chronic lymphocytic leukemia cells. PNAS 2011; 108: 13253-13257.

18. Tang QL, Zhao ZQ, Li JC, Liang Y, Yin JQ, Zou CY et al. Salinomycin inhibits osteosarcoma by targeting its tumor stem cells. Cancer Lett 2011; 11: 13-121.

19. Tai MH, Chang CC, Kiupel M, Webster JD, Olson LK, Trosko JE. Oct4 expression in adult human stem cells: evidence in support of the stem cell theory of carcinogenesis. Carcinogenesis 2005; 26: 495-502.

20. Hadnagy A, Gaboury L, Beaulieu R, Balicki D. SP analysis may be used to identify cancer stem cell populations. Exp Cell Res 2006; 312: 3701-3710.

21. Donnenberg VS, Donnenberg AD. Multiple drug resistance in cancer revisited: the cancer stem cell hypothesis. J Clin Pharmacol 2005; 5: 872-877.

22. Tetsu $\mathrm{O}$, McCormick F. Beta-catenin regulates expression of cyclin D1 in colon carcinoma cells. Nature 1999; 398: 422-446.

23. Burstyn-Cohen T, Stanleigh J, Sela-Donenfeld D, Kalcheim C. Canonical Wnt activity regulates trunk neural crest delamination linking BMP/noggin signaling with $\mathrm{G} 1 / \mathrm{S}$ transition. Development 2004; 131: 5327-5339.

24. Rimerman RA, Gellert-Randleman A, Diehl JA. Wnt1 and MEK1 cooperate to promote cyclin D1 accumulation and cellular transformation. J Biol Chem 2000; 275: 14736-14742.

25. Megason SG, Andrew PM. A mitogen gradient of dorsal midline Wnts organizes growth in the CNS. Development 2002; 12: 2087-2098.

26. Cai $\mathrm{C}$, Zhu $\mathrm{X}$. The $\mathrm{Wnt} / \beta$-catenin pathway regulates self-renewal of cancer stem-like cells in human gastric cancer. Mol Med Rep 2012; 5: 1191-1196.

27. Kanwar SS, Yu Y, Nautiyal J, Patel BB, Majumdar AP. The Wnt/beta-catenin pathway regulates growth and maintenance of colonospheres. Mol Cancer 2010; 9: 212.

28. Vermeulen L, Melo DSE, Van Der Heijden M, Cameron K, de Jong JH, Borovski T et al. Wnt activity defines colon cancer stem cellsand is regulated by the microenvironment. Nat Cell Biol 2010; 12: 468-476.

29. Corbo C, Orrù S, Gemei M, Noto RD, Mirabelli P, Imperlini E et al. Protein cross-talk in $\mathrm{CD} 133$ + colon cancer cells indicates activation of the Wnt pathway and upregulation of SRp20 that is potentially involved in tumorigenicity. Proteomics 2012; 12: 2045-2059.

30. Shenoy AK, Fisher RC, Butterworth EA, Pi L, Chang LJ, Appelman HD et al. Transition from colitis to cancer: high Wnt activity sustains the tumor-initiating potential of colon cancer stem cell precursors. Cancer Res 2012; 72: 5091-5100.

31. Nichols J, Zevnik B, Anastassiadis K, Niwa H, Nebenius DK, Chamber I et al. Formation of pluripotent stem cells in the mammalian embryo depends on the POU transcription factor Oct4. Cell 1998; 95: 379-931.

32. Babaie Y, Herwig R, Greber B, Brink TC, Wruck W, Groth D et al. Analysis of Oct4-dependent transcriptional networks regulating self-renewal and pluripotency in human embryonic stem cells. Stem Cells 2007; 25: 500-510.

33. Hochedlinger K, Yamada Y, Beard C, Jaenisch R. Ectopic expression of Oct-4 blocks progenitor-cell differentiation and causes dysplasia in epithelial tissues. Cell 2005; 121: 465-477.

34. Jinsuk K, Arvind S, Dean T. Stem cells, stress, metabolism and cancer: a drama in two Octs. Cell 2009; 34: 491-499.

35. Ezeh UI, Turek PJ, Reijo RA, Clark AT. Human embryonic stem cell genes OCT4, NANOG, STELLAR and GDF3 are expressed in both seminoma and breast carcinoma. Cancer 2005; 104: 2255-2265.

36. Hu T, Liu S, Breiter DR, Wang F, Tang Y, Sun S. Octamer 4 small interfering RNA results in cancer stem cell-like cell apoptosis. Cancer Res 2008; 68: 6533-6540.

37. Tai MH, Chang CC, Olson LK, Trosko JE. Oct4 expression in adult human stem cells: evidence in support of the stem cell theory of carcinogenesis. Carcinogenesis 2005; 26: 495-502.

38. Steingart RA, Heldenberg E, Pinhasov A, Brenneman DE, Fridkin M, Gozes I. A vasoactive intestinal peptide receptor analog alters the expression of homeobox genes. Life Sci 2002; 71: 2543-2552.

39. Atlasi Y, Mowla SJ, Ziaee SA, Bahrami AR. OCT-4, an embryonic stem cell marker, is highly expressed in bladder cancer. Int J Cancer 2007; 120: 1598-1602.

40. Liu J, Ma L, Xu J, Liu C, Zhang J, Chen R et al. Spheroid body-forming cells in the human gastric cancer cell line MKN-45 possess cancer stem cell properties. Int J Oncol 2013; 42: 453-459.

41. Kelly KF, Ng DY, Jayakumaran G, Wood GA, Koide H, Doble BW. $\beta$-catenin enhances Oct4 activity and reinforces pluripotency through a TCF-independent mechanism. Cell Stem Cell 2011; 8: 214-227.

42. Miki T, Yasuda S, Kahn M. Wnt/ $\beta$-catenin signaling in embryonic stem cell self-renewal and somatic cell reprogramming. Stem Cell Rev Rep 2011; 7: 836-846.

43. Hochedlinger K, Yamada Y, Beard C, Jaenisch R. Ectopic expression of Oct-4 blocks progenitor-cell differentiation and causes dysplasia in epithelial tissues. Cell 2005; 121: 465-477. 
44. Dean M, Fojo T, Bates S. Tumour stem cells and drug resistance. Nat Rev Cancer 2005; 5 275-284.

45. Takahashi-Yanaga F, Sasaguri T. The Wnt/ $\beta$-catenin signaling pathway as a target in drug discovery. J Pharmacol Sci 2007; 4: 293-302.

46. Zhang Y, Wang Z, Yu J, Shi JZ, Wang C, Fu WH et al. Cancer stem-like cells contribute to cisplatin resistance and progression in bladder cancer. Cancer Lett 2012; 322: 70-77.
Cell Death and Disease is an open-access journal published by Nature Publishing Group. This work is licensed under a Creative Commons Attribution-NonCommercialShareAlike 3.0 Unported License. To view a copy of this license, visit http://creativecommons.org/licenses/by-nc-sa/3.0/

Supplementary Information accompanies this paper on Cell Death and Disease website (http://www.nature.com/cddis) 